\title{
Carotenoids and their geometry isomers in selected tropical fruits
}

\begin{abstract}
This study determined the carotenoids content in cherry tomato, pink guava, and red grapefruit pulps and juices. Cherry tomato pulp exhibited the highest $\beta$-carotene content whereas pink guava pulp had the highest lycopene content. However, $\beta$-carotene and lycopene contents in the studied fruit juices were lower than their pulps in the same sample portion. Interestingly, six to twelve cis-isomers of carotenoids were identified in the fruit pulps and juices studied. A higher number of trace amounts of cis-carotenoids was found in fruit pulps as compared to juices. Therefore, consumption of whole fruit is recommended as the studied fruit juices have lower carotenoids content.
\end{abstract}

Keyword: Cherry tomato; Red grapefurit; Pink guava; $\beta$-Carotene; Lycopene 